\title{
Creating and Scripting Second Life Bots Using MPML3D
}

\author{
Birgit Endrass ${ }^{1,2}$, Helmut Prendinger ${ }^{1}$, Elisabeth Andrée ${ }^{2}$, \\ and Mitsuru Ishizuka ${ }^{3}$ \\ ${ }^{1}$ National Institute of Informatics, Tokyo, Japan \\ ${ }^{2}$ University of Augsburg, Germany \\ ${ }^{3}$ University of Tokyo, Japan
}

\section{Motivation}

The use of virtual characters becomes invariably common in computer games and other applications. So-called "bots" (computer-controlled virtual characters), may explain virtual settings in a natural way. We describe an example scenario in the online world Second Life [1] in order to explain to content creators how to create bots and script their behavior using the well established authoring language MPML3D [2]. MPML3D does not assume knowledge of a high-level programming language. In Second Life users are represented by their avatars (virtual characters controlled by humans) and its environment provides character models, animations and a graphics engine.

\section{Example Scenario in SL}

Our example scenario contains a poster session where users can be informed about NII (National Institute of Informatics) projects. It involves two bots (see Fig. 1) that explain the setting. When a user, i.e., his or her avatar, approaches the scene, the bots will perceive the avatar, adjust their position and start the presentation.

The virtual character's appearance can be chosen from several predefined templates. To create individual avatars Second Life provides character modifications as well as the possibility to upload user-created objects (like clothes or body parts). Additionally, appearances or items can be bought in online marketplaces and "attached" to the character.

To control the bots' behavior, we use the authoring language MPML3D. The head section of the MPML3D script provides information about the entities. For the example scenario, two bots (Yuki and Ken) are loaded into the scene.

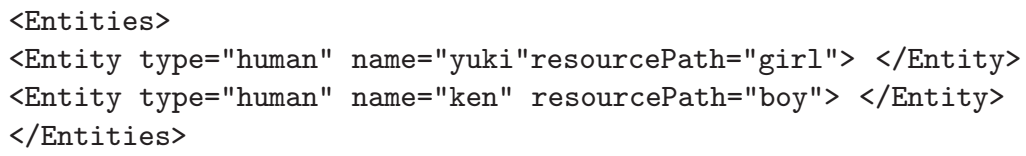




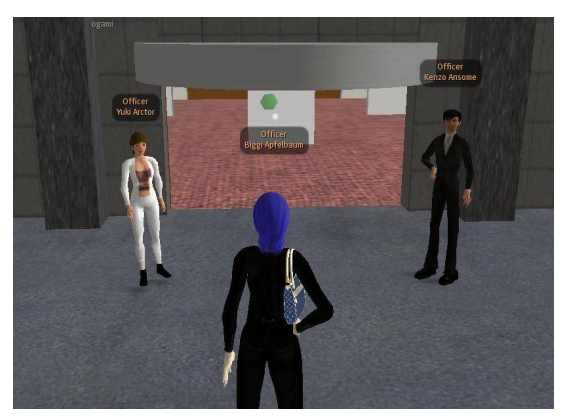

Fig. 1. Screenshot of example scenario in Second Life with two bots (left \& right) and an user avatar (center)

The body part of the MPML3D script defines the bots' actions (verbal and nonverbal behavior. In the example below the female bot (Yuki) performs a sentence accompanied by a pointing gesture (while speaking the 6th word of the sentence (i.e., "this")). Behavior of different bots does not have to be sequential. In the example Kenzo will perform a head nod while he is introduced by Yuki.

<Parallel>

$<$ Action name="yukiSpeak">yuki.speak("My name is Yuki. And this is my coworker Kenzo.")</Action>

<Action startOn="yukiSpeak [6] . begin">yuki.gesture ("POINT_LEFT")</Action>

$<$ Action startOn="yukiSpeak[10] .begin">ken.gesture ("AGREE")</Action>

</Parallel>

SL provides a large set of gestures. Additional animations can be uploaded to Second Life in the commonly used BVH motion file format.

Our approach combines the following features: (1) Simplicity: no previous knowledge in modeling or in high-level programming languages is required, (2) Reusability: existing animations can be easily converted and reused in SL, (3) Low cost: all tools used in this approach are available for free, (4) Community: using Second Life as a platform a large community of users can be reached.

Other approaches in this area do either not provide an authoring tool for easy scripting of agent behavior or do not use free online worlds as a platform.

Acknowledgements. The first author was supported by an International Internship Grant from NII under a Memorandum of Understanding with the University of Augsburg and a Grant from the Elitenetzwerk Bayern (Elite Network Bavaria).

\section{References}

1. http://secondlife.com/ (last viewed: 07.04.2008)

2. Nischt, M., Prendinger, H., André, E., Ishizuka, M.: MPML3D: a reactive framework for the multimodal presentation markup language. In: Procedings of 6th International Conference on Intelligent Virtual Agents, pp. 218-229 (2006) 